\title{
Spatial-Temporal Water-Filling Power Allocation in MIMO Systems with Harvested Energy
}

\author{
Congshi Hu, Jie Gong, Xiaolei Wang, Sheng Zhou, Zhisheng Niu \\ Tsinghua National Laboratory for Information Science and Technology, \\ Dept. of Electronic Engineering, Tsinghua Univ., Beijing, 100084, P. R. China \\ \{hucs11, gongj08, wang-x109\}@ mails.tsinghua.edu.cn, \{sheng.zhou, niuzhs\}@ tsinghua.edu.cn
}

\begin{abstract}
This paper presents the power allocation solution in single link Multi-Input Multi-Output (MIMO) wireless communication systems powered by harvested energy. We first derive the channel capacity with non-causal full information in a finite time horizon, which is shown to be a convex optimization problem. The optimal power allocation can be obtained through SpatialTemporal Water-Filling (ST-WF) solution, by which harvested energy is first allocated among antennas and then among time slots. For the causal information condition, we propose three suboptimal policies, namely constant water level policy, capacityaware water level policy and energy-aware water level policy. Their performance is evaluated by numerical simulations, which show that the energy-aware water level policy is the best among them.
\end{abstract}

\section{INTRODUCTION}

Recently, energy harvesting has received much attention in wireless communication systems because of its environmental friendship and convenient deployment. In such systems, transmitters can harvest energy from random energy resources, such as sun or wind. However, the harvested energy varies both in the time domain and the space domain [1], which leads to new challenges for resource management. For instance, the Multi-Input Multi-Output (MIMO) antenna system has been widely used in modern communications, but how to utilize the harvested energy efficiently in the MIMO system is still an open issue.

In the literature, there have been a lot of researches on energy harvesting issues and the power allocation is one of the hottest topics. For the causal information condition, power allocation policies of energy harvesting are studied in [2]- [4] using Markov decision process (MDP) approach. In [2], the energy allocation and control problem is analyzed for communication satellites. The cross-layer resource management is studied in [3]. Ref. [4] considers the energy allocation policy with stability in sensor networks. Although these causal information based policies are proposed, the properties of the optimal solution cannot be directly obtained. Recently, some research efforts have been done to study the structure of the optimal power allocation based on the non-causal full information condition [5]- [8]. In [5], the

The research work is partially sponsored by the National Basic Research Program of China (973Green: No. 2012CB316001); by the Nature Science Foundation of China (No. 61201191, No. 61021001, No. 60925002); and by Hitachi R\&D Headquarter. authors present the optimal transmission policy to maximize the short-term throughput with finite battery capacity and find the relation between the throughput maximization problem and the completion time minimization problem. Refs. [6][8] consider using directional water-filling power allocation algorithm in different channel models including the Gaussian fading channel [6], the broadcast channel [7] and the multiple access channel [8] to maximize the throughput.

In this paper, we begin with discussing the throughput maximization problem in the energy harvesting MIMO system under the non-causal full information condition, which means the transmitter knows all the future channel states and harvested energy amounts. We show that it is a convex optimization problem and thus the global optimal solution can be achieved. Considering the energy causality, which means energy can be used only after its arrival, and the channel fading in the MIMO system, we propose the Spatial-Temporal Water-Filling (ST-WF) solution, which is proved to be the optimal solution under the non-causal full information condition. For the STWF solution, power is allocated in the spatial domain first and then in the temporal domain. Specifically, a constant energy level is set for different antennas in the same slot. While for the water levels in different slots, the directional water-filling is used for optimization. Note that, however, these two parts are not completely separated, because the allocated power in a given slot is not only affected by the inflow and outflow energy, but also decided by the channel fading of each antenna. In addition, based on the insights from the ST-WF solution, we propose three suboptimal causal information based policies to decide water level slot by slot for practical application.

The rest of the paper is organized as follows. Section II presents the system model. The optimal non-causal full information based solution for maximizing throughput in the energy harvesting MIMO system is studied in Section III. The causal information based policies will be proposed in Section IV, followed by simulation results in Section V. And finally, Section VI concludes this work. For future use, we introduce the key concepts and terminologies in Table I.

\section{SySTEM MODEL}

We consider a single wireless link as shown in Fig. 1, where the transmitter and the receiver are equipped with $n_{t}$ and $n_{r}$ antennas, respectively. The transmitter is powered by the energy harvested from random energy sources, such as 
TABLE I

PARAMETERS IN THE PAPER

\begin{tabular}{|l|l|}
\hline Notations & Definition \\
\hline $\mathbb{C}$ & Set of complex numbers \\
$\operatorname{tr}(\mathbf{A})$ & Trace of matrix $\mathbf{A}$ \\
$\mathbf{A}^{\mathrm{H}}$ & Conjugate transpose matrix of $\mathbf{A}$ \\
$n_{t}$ & Number of transmit antennas \\
$n_{r}$ & Number of receive antennas \\
$\mathbf{X}(j)$ & Transmit signal vector in slot $j$ \\
$\mathbf{Y}(j)$ & Receive signal vector in slot $j$ \\
$\mathbf{H}(j)$ & Channel gain matrix in slot $j$ \\
$\mathbf{Q}(j)$ & The auto-covariance of $\mathbf{X}(j)$ \\
$p_{m}(j)$ & Power allocated to antenna $m$ in slot $j$ \\
$E_{j}$ & Harvested energy in time slot $j$ \\
$\lambda_{m}(j)$ & The $m$ th singular value of $\mathbf{H}(j)$ \\
$\mathbf{U}$ & Eigenmatrix of $\mathbf{H}^{\mathrm{H}} \mathbf{H}$ \\
\hline
\end{tabular}

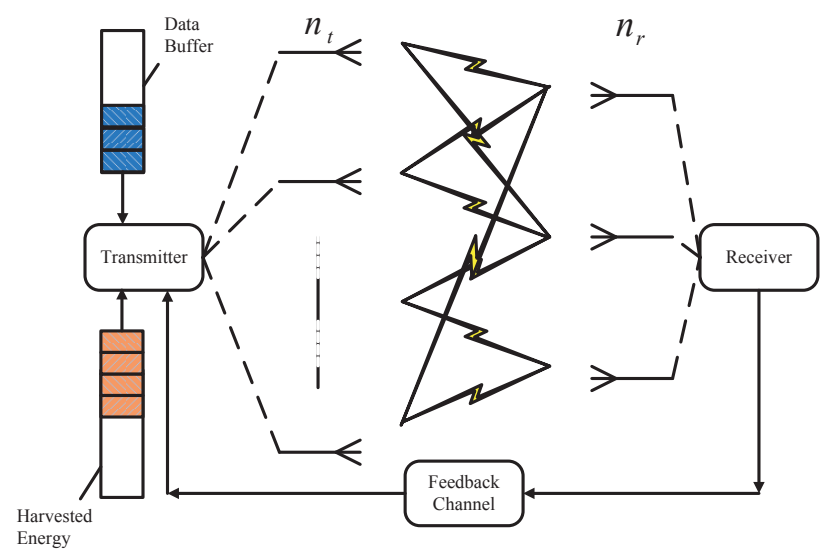

Fig. 1. The energy harvesting MIMO system model.

solar energy. The harvested energy is stored in a battery whose capacity is $E_{\max }$. We assume that the harvested energy is used only for transmission, which means that the processing energy is ignored. The receive signal vector $\mathbf{Y}(t) \in \mathbb{C}^{n_{r} \times 1}$ at time $t$ is

$$
\mathbf{Y}(t)=\mathbf{H}(t) \mathbf{X}(t)+\mathbf{n}(t)
$$

$\mathbf{H}(t)$ is the $n_{r} \times n_{t}$ channel state matrix and $\mathbf{X}(t) \in \mathbb{C}^{n_{t} \times 1}$ is the transmit signal vector. $\mathbf{n}(t)$ is additive white Gaussian noise with zero mean and unit variance, i.e.,

$$
\mathrm{E}\left[\mathbf{n}(t) \mathbf{n}(t)^{\mathrm{H}}\right]=\mathbf{I}_{n_{r}} .
$$

The mutual information is given by [9]

$$
\mathrm{I}(\mathbf{X}(t) ; \mathbf{Y}(t))=\log \operatorname{det}\left[\mathbf{I}_{n_{r}}+\mathbf{H}(t) \mathbf{Q}(t) \mathbf{H}^{\mathrm{H}}(t)\right],
$$

where $\mathbf{Q}(t)$ is the auto-covariance of transmitted signal vector, i.e.,

$$
\mathrm{E}\left[\mathbf{X}(t) \mathbf{X}^{\mathrm{H}}(t)\right]=\mathbf{Q}(t) .
$$

We consider a time-slotted system with slot length $T_{f}$ and study the power allocation in a finite time horizon, i.e., $N$ slots. The harvested energy $\left\{E_{0}, E_{1}, \ldots, E_{N-1}\right\}$ arrives at the beginning of each slot. In slot $j$, the transmission power is denoted as $p_{j}$, i.e.,

$$
\operatorname{tr}\left[\mathbf{X}(j) \mathbf{X}^{\mathrm{H}}(j)\right]=p_{j} .
$$

And the power allocated to antenna $m$ in slot $j$ is denotes as $p_{m}(j)$, which is also the $m$ th diagonal element of $\mathbf{Q}(j)$.

There are two main constraints on energy harvesting, namely the energy causality constraint and the battery capacity constraint. For the causality, the harvested energy cannot be consumed before its arrival, which can be expressed as

$$
\sum_{j=1}^{k} T_{f} p_{j} \leq \sum_{j=0}^{k-1} E_{j}, k=1,2, \ldots, N
$$

Note that the constraint in (6) must be satisfied with equality when $k=N$ for optimization. Otherwise, we can always get higher throughput by increasing the power $p_{N}$ without conflicting any constraint. The other constraint is that the harvested energy cannot exceed the battery capacity, i.e.,

$$
\sum_{j=0}^{k} E_{j}-\sum_{j=1}^{k} T_{f} p_{j} \leq E_{\max }, k=1,2, \ldots, N-1 .
$$

\section{Throughput Maximization With Non-CAUSAL FULL INFORMATION}

\section{A. Problem Formulation}

For the non-causal full information condition, the transmitter knows future energy arrivals and channel states of all the $N$ slots before the transmission. The problem is written as

Problem A:

$$
\begin{array}{cr}
\max & \sum_{j=1}^{N} \frac{T_{f}}{2} \log \operatorname{det}\left[\mathbf{I}_{n_{r}}+\mathbf{H}(j) \mathbf{Q}(j) \mathbf{H}^{\mathrm{H}}(j)\right] \\
\text { s.t. } & \sum_{j=1}^{k} T_{f} p_{j} \leq \sum_{j=0}^{k-1} E_{j}, \forall k \leq N, \\
\sum_{j=0}^{k} E_{j}-\sum_{j=1}^{k} T_{f} p_{j} & \leq E_{\max }, \forall k \leq N-1, \\
p_{m}(j) & \geq 0, \forall j \leq N .
\end{array}
$$

Using the determinant identity [11] $\operatorname{det}(\mathbf{I}+\mathbf{A B})=$ $\operatorname{det}(\mathbf{I}+\mathbf{B A})$, the mutual information is shown as

$$
I(\mathbf{X} ; \mathbf{Y})=\log \operatorname{det}\left(\mathbf{I}+\mathbf{H Q} \mathbf{H}^{\mathrm{H}}\right)=\log \operatorname{det}\left(\mathbf{I}+\mathbf{Q} \mathbf{H}^{\mathrm{H}} \mathbf{H}\right) .
$$

Since $\mathbf{H}^{\mathrm{H}} \mathbf{H}$ is Hermitian, it can be diagonalized, i.e. ,

$$
\mathbf{H}^{\mathrm{H}} \mathbf{H}=\mathbf{U}^{\mathrm{H}} \Lambda \mathbf{U}
$$

with unitary $\mathbf{U}$ and non-negative diagonal matrix $\boldsymbol{\Lambda}=$ $\operatorname{diag}\left(\lambda_{1}, \ldots, \lambda_{M}, 0, \ldots, 0\right)$, where $M=\min \left(n_{t}, n_{r}\right)$.

The objective function (8) is equivalent to

$$
\max \sum_{j=1}^{N} \frac{T_{f}}{2} \log \operatorname{det}\left[\mathbf{I}+\boldsymbol{\Lambda}^{\frac{1}{2}}(j) \mathbf{U}(j) \mathbf{Q}(j) \mathbf{U}^{\mathrm{H}} \boldsymbol{\Lambda}^{\frac{1}{2}}(j)\right] \text {. }
$$

We denote $\mathbf{U Q U} \mathbf{U}^{\mathrm{H}}$ by $\widetilde{\mathbf{Q}}$ and observe that $\widetilde{\mathbf{Q}}$ is positivesemidefinite and that $\operatorname{tr}(\mathbf{Q})=\operatorname{tr}(\widetilde{\mathbf{Q}})$ since $\mathbf{U}$ is unitary and $\mathbf{Q}$ is positive-semidefinite. In addition, since $\widetilde{\mathbf{Q}}$ is a congruent 
matrix of $\mathbf{Q}, \widetilde{\mathbf{Q}}$ has nonnegative diagonal elements as $\mathbf{Q}$. Consequently, the problem can be reformulated as

Problem B:

$$
\begin{gathered}
\max _{\forall \widetilde{\mathbf{Q}}(j) \in \Omega} \sum_{j=1}^{N} \log \operatorname{det}\left[\mathbf{I}_{n_{t}}+\boldsymbol{\Lambda}^{\frac{1}{2}}(j) \widetilde{\mathbf{Q}}(j) \boldsymbol{\Lambda}^{\frac{1}{2}}(j)\right] \\
\text { s.t. } \quad \sum_{j=1}^{k} T_{f} \operatorname{tr}[\widetilde{\mathbf{Q}}(j)] \leq \sum_{j=0}^{k-1} E_{j}, \forall k, \\
\qquad \sum_{j=0}^{k} E_{j}-\sum_{j=1}^{k} T_{f} \operatorname{tr}[\widetilde{\mathbf{Q}}(j)] \leq E_{\max }, k \leq N-1,(14) \\
\quad-\tilde{q}_{i i}(j) \leq 0, \forall i, \forall j .
\end{gathered}
$$

where $\tilde{q}_{i i}(j)$ is the $i$ th diagonal element of $\widetilde{\mathbf{Q}}(j)$, and $\Omega$ is the set of all the Hermitian matrices.

\section{B. Optimal Non-Causal Full Information Based Solution}

Theorem 1. The Problem $\boldsymbol{B}$ is a convex optimization problem.

Proof: The proof is given in Appendix $A$.

Based on Theorem 1, we can use Lagrangian function for any multipliers $\alpha_{k} \geq 0, \beta_{k} \geq 0, \gamma_{i j} \geq 0$ to solve the problem as

$$
\begin{aligned}
\mathcal{L} & =\sum_{j=1}^{N} \frac{T_{f}}{2} \log \operatorname{det}\left[\mathbf{I}_{n_{t}}+\boldsymbol{\Lambda}^{\frac{1}{2}}(j) \widetilde{\mathbf{Q}}(j) \boldsymbol{\Lambda}^{\frac{1}{2}}(j)\right] \\
& -\sum_{k=1}^{N} \alpha_{k}\left\{\sum_{j=1}^{k} T_{f} \operatorname{tr}[\widetilde{\mathbf{Q}}(j)]-\sum_{j=0}^{k-1} E_{j}\right\} \\
& -\sum_{k=1}^{N} \beta_{k}\left\{\sum_{j=0}^{k} E_{j}-\sum_{j=1}^{k} T_{f} \operatorname{tr}[\widetilde{\mathbf{Q}}(j)]-E_{\max }\right\} \\
& +\sum_{j=1}^{N} \sum_{i=1}^{n_{t}} \gamma_{i j} \tilde{q}_{i i}(j)
\end{aligned}
$$

Additional complementary slackness conditions are as follows,

$$
\begin{aligned}
\alpha_{k}\left\{\sum_{j=1}^{k} T_{f} \operatorname{tr}[\widetilde{\mathbf{Q}}(j)]-\sum_{j=0}^{k-1} E_{j}\right\} & =0, k \leq N-1, \\
\beta_{k}\left\{\sum_{j=0}^{k} E_{j}-\sum_{j=1}^{k} T_{f} \operatorname{tr}[\widetilde{\mathbf{Q}}(j)]-E_{\max }\right\} & =0, k \leq N-1, \\
\gamma_{i j} \tilde{q}_{i i}(j) & =0, \forall i, \forall j .
\end{aligned}
$$

Not that in (17), $k=N$ is not included since this constraint is satisfied with equality as mentioned before.

For the matrix differential calculation, there are two facts [11]. First, the differential of matrix $\mathbf{X}$ 's determinant $|\mathbf{X}|$ is

$$
\mathrm{d}|\mathbf{X}|=|\mathbf{X}| \operatorname{tr}\left(\mathbf{X}^{-1} \mathrm{~d} \mathbf{X}\right)=\operatorname{tr}\left(|\mathbf{X}| \mathbf{X}^{-1} \mathrm{~d} \mathbf{X}\right) .
$$

For a differentiable function $f(\mathbf{X})$, there is

$$
\mathrm{d} f(\mathbf{X})=\operatorname{tr}\{\mathbf{A d} \mathbf{X}\}
$$

if and only of

$$
f(\mathbf{X})=\frac{\mathrm{d} f(\mathbf{X})}{\mathrm{d} \mathbf{X}}=\mathbf{A}^{\mathrm{T}} .
$$

Then by setting $\partial \mathcal{L} / \partial \widetilde{\mathbf{Q}}(j)=\mathbf{0}$, based on Eq. (20), we have

$$
\begin{aligned}
& \mathrm{d}\left\{\log \operatorname{det}\left[\mathbf{I}_{n_{t}}+\boldsymbol{\Lambda}^{\frac{1}{2}}(j) \widetilde{\mathbf{Q}}(j) \boldsymbol{\Lambda}^{\frac{1}{2}}(j)\right]\right\} \\
& =\frac{\{\operatorname{det}[\boldsymbol{\Psi}(j)]\}}{\operatorname{det}[\boldsymbol{\Psi}(j)]} \operatorname{tr}\left[\boldsymbol{\Psi}^{-1}(j) \boldsymbol{\Lambda}^{\frac{1}{2}}(j) \mathrm{d} \widetilde{\mathbf{Q}}(j) \boldsymbol{\Lambda}^{\frac{1}{2}}(j)\right] \\
& =\operatorname{tr}\left[\boldsymbol{\Lambda}^{\frac{1}{2}}(j) \boldsymbol{\Psi}^{-1}(j) \boldsymbol{\Lambda}^{\frac{1}{2}}(j) \mathrm{d} \widetilde{\mathbf{Q}}(j)\right],
\end{aligned}
$$

where $\boldsymbol{\Psi}(j)=\left[\mathbf{I}_{n_{r}}+\boldsymbol{\Lambda}^{\frac{1}{2}}(j) \widetilde{\mathbf{Q}}(j) \boldsymbol{\Lambda}^{\frac{1}{2}}(j)\right]$.

According to Eqs. (21)-(22), we know that

$$
\begin{array}{r}
\partial \mathcal{L} / \partial \widetilde{\mathbf{Q}}(j)=\boldsymbol{\Lambda}^{\frac{1}{2}}(j) \boldsymbol{\Psi}^{-1}(j) \boldsymbol{\Lambda}^{\frac{1}{2}}(j)-\sum_{k=j}^{N} \alpha_{k} \mathbf{I}_{n_{t}}+ \\
\sum_{k=j}^{N-1} \beta_{k} \mathbf{I}_{n_{t}}+\sum_{i=1}^{n_{t}} \gamma_{i j} \mathbf{E}_{i i}=\mathbf{0},
\end{array}
$$

where $\mathbf{E}_{i i}$ denotes the matrix whose elements are all zero except for the $i$ th diagonal element to be one.

From Eq. (24), because the matrix $\boldsymbol{\Lambda}(j)$ is a diagonal matrix, after some manipulation we know that $\widetilde{\mathbf{Q}}(j)$ must be diagonal for the first $M$ columns, i.e.,

$$
\widetilde{\mathbf{Q}}(j)=\operatorname{diag}\left(\tilde{q}_{1}(j), \ldots, \tilde{q}_{M}(j), 0, \ldots, 0\right),
$$

where

$$
\tilde{q}_{m}(j)=\frac{1}{\sum_{i=j}^{N} \alpha_{i}-\sum_{i=j}^{N-1} \beta_{i}-\gamma_{i j}}-\frac{1}{\lambda_{m}(j)}
$$

From (19), we have the optimal power solution as

$$
\tilde{q}_{m}^{*}(j)=\left[\frac{1}{\sum_{i=j}^{N} \alpha_{i}-\sum_{i=j}^{N-1} \beta_{i}}-\frac{1}{\lambda_{m}(j)}\right]^{+},
$$

where $[x]^{+}=\max \{x, 0\}$, followed by the optimal autocovariance matrix as

$$
\mathbf{Q}^{*}(j)=\mathbf{U}^{\mathrm{H}}(j) \widetilde{\mathbf{Q}}^{*}(j) \mathbf{U}(j),
$$

where $\widetilde{\mathbf{Q}}^{*}(j)=\operatorname{diag}\left(\tilde{q}_{1}^{*}(j), \tilde{q}_{2}^{*}(j), \ldots, \tilde{q}_{M}^{*}(j), 0, \ldots, 0\right)$.

Actually Eq. (27) has a very clear physical meaning that it represents the power allocation to $M$ parallel channels according to singular vector directions of the channel matrix H. From Eq. (28), $\mathbf{Q}^{*}$ is the unitary transformation of $\widetilde{\mathbf{Q}}^{*}$. And the unitary transformation does not change the trace, or in other words, power. From another point of view, it is a affine transformation between singular vector directions (virtual parallel channel directions) and actual channel directions.

\section{Spatial-Temporal Water-Filling Algorithm}

In this subsection, we discuss the optimal power allocation and present an intuitive algorithm called Spatial-Temporal Water-Filling (ST-WF). Without loss of generality, we discuss $\widetilde{\mathbf{Q}}^{*}$ here instead of $\mathbf{Q}^{*}$. 
From Eq. (27), we can see that in different antennas in the same slot $j$, they have the same water level, $\frac{1}{\sum^{N} \alpha_{i}-\sum^{N-1} \beta_{i}}$. So the traditional Water-Filling [10] can be used once the total power in the slot is known.

On the other hand, for different time slots, we can see that Eq. (27) has a very similar expression as the single antenna power allocation scenario in [6]. Here, we pick up the main idea called directional water-filling [6], where the author proposed a concept called the right permeable tap between two adjacent slots. It allows energy flow only from left to right which implies the energy causality. And on the other hand, the transferred energy cannot be larger than $E_{\max }$ minus the harvested energy of the next slot due to the battery capacity constraint.

The main contribution of this work is that we extend the power allocation problem to both the spatial domain and the temporal domain. We propose the optimal non-causal full information based power allocation for the energy harvesting MIMO system , namely Spatial-Temporal Water-Filling. First, we allocate power in each slot without considering energy flows, which means we use up all the energy harvested in the slot. According to Eq. (27), the optimal power allocation for each antenna is achieved by the traditional water-filling algorithm. Thus, each slot can get an initial water level. Then, we consider energy flows in the temporal domain. Once the water level in slot $j$ is higher than slot $j+1$, energy can flow from slot $j$ to slot $j+1$. Energy cannot flow in the opposite direction due to causality. Besides, energy cannot flow too much in case of battery overflow. Note that the new water level in each slot is not equal to its initial level plus the water level inflows and minus the water level outflows due to the fading variance among different antennas. In other words, the initial water level helps to decide whether there is energy to flow but cannot directly decide the accurate optimal level. We must reallocate the total new energy flowing from other slots by traditional WF again for optimization. And that is the reason why the spatial domain and the temporal domain are not completely separate.

Examples with 4 transmitting antennas during 2 slots are shown below, which is sufficient to present our idea and easy to extend to more complicated scenarios. The fading levels for each time slot and each antenna is shown in Fig. 2(a), which is a 3-dimension figure presenting both spatial and temporal fading.

We first illustrate an example with lower energy in the first slot. We allocate the harvested energy in each slot using traditional WF as shown in Fig. 2(b). Antenna 3 in the first slot and antenna 1 in the second slot get no power because of deep fading. Comparing the water levels, the second slot is higher. So the optimal power allocation is exactly the same as Fig. 2(b) because energy cannot flow to the previous slot.

Then the second energy condition is a little higher water level in the first slot as Fig. 2(c). So the water can flow to the next slot and the total water is not too much to make battery overflow in the second slot. As a result, these two water levels are the same, which is shown in Fig. 2(d). Note that antenna 1 in the second slot gets power after energy balancing though it got nothing in the initial allocation.

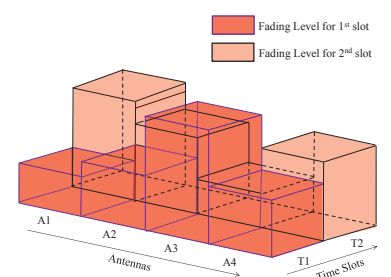

(a) Fading states for the examples

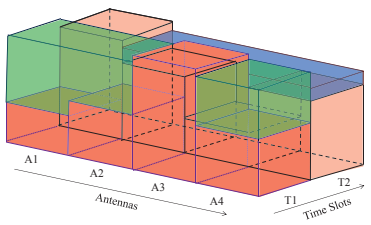

(c) Higher water level at first

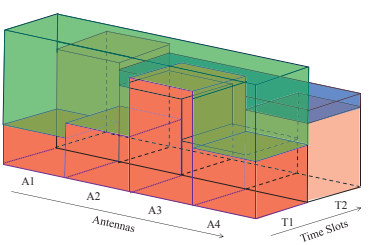

(e) Much higher water level at first

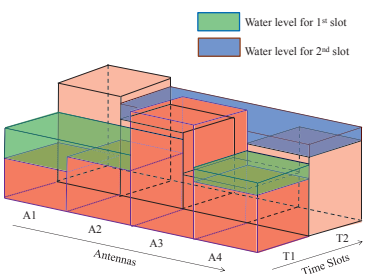

(b) Low water level at first

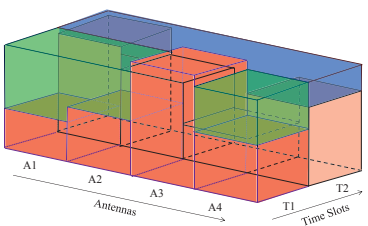

(d) Equal levels after optimization

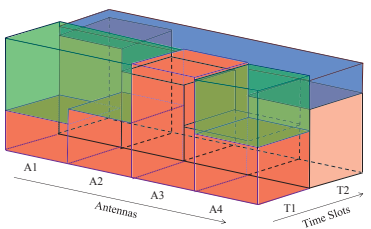

(f) Unequal levels after optimization
Fig. 2. Different water level conditions for ST-WF operation.

At last, we give the third example in Fig. 2(e)-2(f). The first slot has much higher level as Fig 2(e). Because of the battery capacity constraint, we cannot outflow too much energy from the first slot to the second slot to make the water levels the same. Thus, there is still a gap between the optimal energy levels as Fig 2(f). Note that the antenna 3 in the first slot gets no power after energy balancing because of its energy outflow and deep fading though it got power initially.

\section{Suboptimal Causal Information Based Policy}

We study the causal information based policies for this throughput maximization problem based on the properties of the ST-WF algorithm. The transmitter knows the battery level and the current channel state, but it has no knowledge about future states like arriving energy and future channel states. The harvested energy follows non-negative uniform distribution [12] with the average energy per slot $E_{\text {ave }}$.

We first show some hints given by the ST-WF solution. If we ignore the energy causality constraint and the battery capacity constraint, the optimal power allocation will be constant after the ST-WF operation for all the slots. Considering the finite battery capacity, we want to avoid from battery overflow and use more energy if there is too much energy left in the battery. Furthermore, because of the randomness of harvested energy 
arrivals, a better way to use the energy is to adapt the power allocation based on the energy condition. Thus, we propose three algorithms accordingly as shown below.

\section{A. Constant Water Level}

Motivated by the fact that the optimal non-causal full information solution tries to achieve a constant water level, we propose this constant water level algorithm. The water level $\frac{1}{\gamma_{0}}$ is calculated by solving

$$
\int_{\gamma_{0}}^{\infty}\left(\frac{1}{\gamma_{0}}-\frac{1}{\gamma}\right) f(\gamma) \mathrm{d} \gamma=\frac{E_{\text {ave }}}{T_{f} n_{t}}
$$

where both sides of the equation are the power expectation for each antenna in each slot.

Then the power of antenna $m$ in slot $j$ is decided by

$$
p_{m}^{\text {Const }}(j)=\left[\frac{1}{\gamma_{0}}-\frac{1}{\lambda_{m}(j)}\right]^{+} .
$$

If the energy in the battery is not enough to support the total power of all antennas, i.e., $\sum_{m=1}^{n_{t}} p_{m}(j)$, then the transmitter keeps silent in this slot.

\section{B. Capacity Aware Water Level}

We propose the second policy to avoid the risk of reaching the capacity. If the expected battery energy of the next slot $j+1$, i.e. , $E_{\text {in }}(j)+E_{\text {ave }}$ is higher the battery capacity, then energy of $E_{\text {in }}(j)+E_{\text {ave }}-E_{\max }$ must be used in the current slot. Here, $E_{\text {in }}(j)$ denotes the battery energy at the beginning of slot $j$. Note that those energy must be allocated to achieve a constant water level for all antennas. We denote this solution as $p_{m}^{\mathrm{C}}(j)$, then the second power allocation is determined as

$$
p_{m}^{\mathrm{CA}}(j)=\max \left\{p_{m}^{\mathrm{C}}(j), p_{m}^{\mathrm{Const}}(j)\right\},
$$

where $p_{m}^{\text {Const }}(j)$ is expressed as Eq. (30). Because this solution considers the battery capacity limitation, it is expected to improve the performance.

\section{Energy Aware Water Level}

Besides battery capacity, another reason why the optimal level is not constant is the energy causality. We want to change the water level according to the current energy condition. At the beginning of time slot $j$, water level is determined by

$$
\int_{\gamma_{0, j}}^{\infty}\left(\frac{1}{\gamma_{0, j}}-\frac{1}{\gamma}\right) f(\gamma) \mathrm{d} \gamma=\frac{(N-j) E_{\text {ave }}+E_{\text {in }}(j)}{(N-j+1) T_{f} n_{t}} .
$$

So the power allocation is decided by

$$
p_{m}^{\mathrm{E}}(j)=\left[\frac{1}{\gamma_{0, j}}-\frac{1}{\lambda_{m}(j)}\right]^{+} .
$$

Also considering the battery capacity, we get the solution as

$$
p_{m}^{\mathrm{EA}}(j)=\max \left\{p_{m}^{\mathrm{E}}(j), p_{m}^{\mathrm{C}}(j)\right\} .
$$

This policy considers both battery capacity and energy condition, so it should further improve the throughput.

\section{Numerical RESUlT}

In this section, we compare the performance of the proposed causal information based policies with the optimal non-causal full information based policy. We use the Rayleigh fading and generate independent random channel matrices for each run. We set 80 time slots and the MIMO system is equipped with 4 transmitting antennas and 4 receiving antennas. A total number of 1000 monte carlo simulation runs are performed for different parameter setups. Besides, the average channel gain is $0 \mathrm{~dB}$. The harvested energy follows non-negative uniform distribution.

We first exam the performance of those algorithms under different average harvested energy. The battery capacity is $5 \mathrm{~J}$, i.e., $E_{\max }=5 \mathrm{~J}$. The numerical results are shown in Fig. 3. It is observed that the energy-aware water level policy performs better than the other two, especially for high average harvested energy. The reason is that when the average harvested energy is higher, it is more likely to be overflow. Thus, adaptively changing the water level can manage the energy usage better. In other words, if there is much energy in the battery, we tend to allocate more power currently and vice versa. From another point of view, the higher probability of overflow for high average harvested energy is also the reason why the gap between the optimal ST-WF policy and causal information policies increases with average harvested energy.

Another test is the performance under different battery capacities as Fig. 4, where the average harvested energy is set to be $2 \mathrm{~J}$ per slot. The energy-aware policy performs best again. Another observation is that the distance between the ST-WF policy and causal information policies decreases as battery capacity increases. This is because that as battery capacity increases, the optimal water level becomes more smooth, which is the idea of causal information based policies.

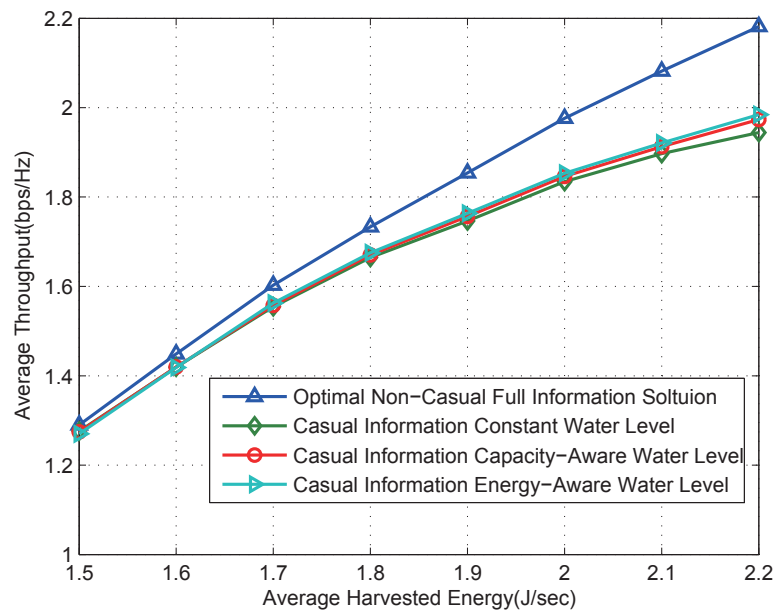

Fig. 3. Performance of the policies for different energy recharge rates.

\section{CONCLUSION}

In this work, we have proposed an optimal power allocation solution called Spatial-Temporal Water-Filling for the energy 


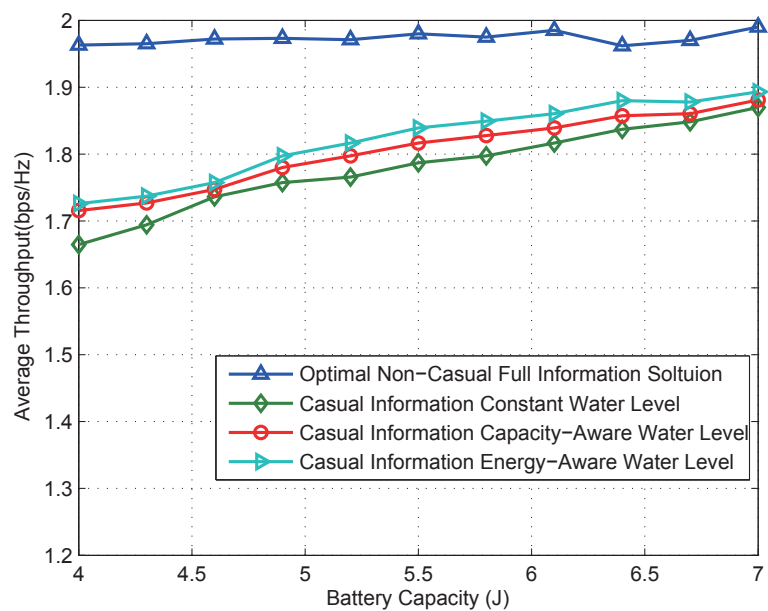

Fig. 4. Performance of the policies for different battery capacities.

harvesting MIMO system with non-causal full information. In addition, motived by the non-causal full information based solution, three causal information based policies have been proposed, namely the constant water level, the capacity-aware water level and the energy-aware water level. Their performance have been evaluated by numerical simulations and we have concluded that the energy-aware water level is the best among them.

\section{APPENDIX}

\section{A. Proof of the Convex Optimality}

To prove Problem $\mathbf{B}$ is a convex optimization problem, we calculate the Hessian matrix of the objective function and the constraints.

Theorem A1. [11] Given a twice differentiable matrix function $f(\mathbf{X})$, there is a relation that

$$
\mathrm{d}^{2} f(\mathbf{X})=\operatorname{tr}\left[\mathbf{B}(\mathrm{d} \mathbf{X})^{\mathrm{H}} \mathbf{C} d \mathbf{X}\right],
$$

if and only if

$$
\mathbf{H}(f(\mathbf{X}))=\frac{1}{2}\left(\mathbf{B}^{\mathrm{H}} \otimes \boldsymbol{C}+\boldsymbol{B} \otimes \mathbf{C}^{\mathrm{H}}\right),
$$

where $\mathbf{H}(f(\mathbf{X}))$ is the Hessian matrix of $f(\mathbf{X})$ and $\otimes$ denotes the Kronecker product.

Another fact is that if $\mathbf{A}$ and $\mathbf{B}$ are Hermitian positivesemidefinite matrices, their Kronecker product $\mathbf{A} \otimes \mathbf{B}$ will be also a Hermitian positive-semidefinite matrix.

Then we calculate the twice differential matrix of Eq. (12). From Eq. (23) we know that

$$
\mathrm{d}[\log \operatorname{det} \boldsymbol{\Psi}(j)]=\operatorname{tr}\left[\boldsymbol{\Lambda}^{\frac{1}{2}}(j) \boldsymbol{\Psi}^{-1}(j) \boldsymbol{\Lambda}^{\frac{1}{2}}(j) \mathrm{d} \widetilde{\mathbf{Q}}(j)\right] .
$$

And the twice differential matrix is calculated as

$$
\begin{aligned}
& \mathrm{d}^{2} \log \operatorname{det}\left[\mathbf{I}_{n_{t}}+\boldsymbol{\Lambda}^{\frac{1}{2}}(j) \widetilde{\mathbf{Q}}(j) \boldsymbol{\Lambda}^{\frac{1}{2}}(j)\right] \\
= & \operatorname{tr}\left\{\boldsymbol{\Lambda}^{\frac{1}{2}}(j) \mathrm{d}\left[\boldsymbol{\Psi}^{-1}(j)\right] \boldsymbol{\Lambda}^{\frac{1}{2}}(j) \mathrm{d} \widetilde{\mathbf{Q}}(j)\right\} \\
= & \operatorname{tr}\left\{\boldsymbol{\Lambda}^{\frac{1}{2}}(j) \Psi^{-1}(j) \mathrm{d}[\boldsymbol{\Psi}(j)] \boldsymbol{\Psi}^{-1}(j) \boldsymbol{\Lambda}^{\frac{1}{2}}(j) \mathrm{d} \widetilde{\mathbf{Q}}(j)\right\} .
\end{aligned}
$$

Since $\widetilde{\mathbf{Q}}(j)$ is Hermitian, i.e., $\widetilde{\mathbf{Q}}^{\mathrm{H}}(j)=\widetilde{\mathbf{Q}}(j)$, it is easy to know that $\mathrm{d} \widetilde{\mathbf{Q}}^{\mathrm{H}}(j)=[\mathrm{d} \widetilde{\mathbf{Q}}(j)]^{\mathrm{H}}=\mathrm{d} \widetilde{\mathbf{Q}}(j)$.

As a result, there is

$$
\mathrm{d}^{2}[\log \operatorname{det} \boldsymbol{\Psi}(j)]=\operatorname{tr}\left\{\boldsymbol{\Theta}(j)[\mathrm{d} \widetilde{\mathbf{Q}}(j)]^{\mathrm{H}} \boldsymbol{\Theta}(j) \mathrm{d} \widetilde{\mathbf{Q}}(j)\right\},
$$

where $\boldsymbol{\Theta}(j)=\boldsymbol{\Lambda}^{\frac{1}{2}}(j) \boldsymbol{\Psi}^{-1}(j) \boldsymbol{\Lambda}^{\frac{1}{2}}(j)$ is a Hermitian positivesemidefinite matrix.

Based on the Theorem A1, the Hessian matrix of (12) is $\boldsymbol{\Theta}(j) \otimes \boldsymbol{\Theta}(j)$, which is a Hermitian positive-semidefinite matrix. So, the objective function (12) is concave.

As for the constraints in (13)-(15), they are linear since their differential matrix is the identity matrix.

Now we know that Problem B is a convex optimization since its objective function is concave and constraints are linear.

\section{REFERENCES}

[1] M. Gorlatova, A. Wallwater, and G. Zussman, "Networking low-powe energy harvesting devices: measurements and algorithms", Proceedings of IEEE International Conference on Computer Communications (INFO COM), Shanghai, pp. 1602-1610, April 2011.

[2] A. C. Fu, E. Modiano, and J. N. Tsitsiklis, "Optimal energy allocation and admission control for communications satellites, IEEE/ACM Trans. networking, Vol. 11, No. 3, Jun. 2003

[3] M. Gatzianas, L. Georgiadis, and L. Tassiulas, "Control of wireless networks with rechargeable batteries, IEEE Trans. Commun., Vol. 9, No. 2, Feb. 2010

[4] V. Sharma, U. Mukherji, V. Joseph, and S. Gupta, "Optimal energy management policies for energy harvesting sensor nodes, IEEE Trans. Wireless Commun., Vol. 9, No. 4, Apr. 2010

[5] K. Tutuncuoglu and A. Yener, Optimum transmission policies for battery limited energy harvesting nodes, IEEE Trans. Commun., Vol. 11, No. 3 , Mar. 2012

[6] O. Ozel, K. Tutuncuoglu, J. Yang, S. Ulukus, and A. Yener, Transmission with energy harvesting nodes in fading wireless channels: optimal policies, IEEE J. Selected Area Commun., Vol. 29, No. 8, Sept. 2011

[7] J. Yang, O. Ozel, and S. Ulukus, "Broadcasting with an energy harvesting rechargeable transmitter," IEEE Trans. on Wireless Commun., Vol. 11, Issue 2, pp. 571-583, February 2012.

[8] J. Yang and S. Ulukus, "Optimal packet scheduling in a multiple access channel with rechargeable nodes", IEEE International Conference on Communications (ICC), Kyoto, June 2011.

[9] E. Telatar, "Capacity of multi-antenna Gaussian channels", AT\&T-Bell Labs Internal Tech. Memo., June 1995.

[10] A. Goldsmith, "Wireless Communications", Cambridge University, 2005.

[11] Xianda. Zhang, "Matrix Analysis and Applications", Tsinghua University Press, Beijing, 2004.

[12] B. Medepally, N. B. Mehta, and C. R. Murthy, "Implications of energy profile and storage on energy harvesting sensor link performance,Proceedings of Global Telecommunications Conference (GLOBECOM),December 2009, pp.1-6. 